\title{
Oxyntomodulin regulates resetting of the liver circadian clock by food
}

\author{
Dominic Landgraf ${ }^{1+\ddagger \S}$, Anthony H Tsang ${ }^{1,2 \dagger}$, Alexei Leliavski ${ }^{1,2}$, Christiane E Koch ${ }^{2}$, \\ Johanna L Barclay', Daniel J Drucker ${ }^{3,4}$, Henrik Oster ${ }^{1,2 *}$
}

${ }^{1}$ Circadian Rhythms Group, Max Planck Institute for Biophysical Chemistry, Göttingen, Germany; ${ }^{2}$ Chronophysiology Group, Medical Department I, University of Lübeck, Lübeck, Germany; ${ }^{3}$ Department of Medicine, University of Toronto, Toronto, Canada; ${ }^{4}$ Lunenfeld-Tanenbaum Research Institute, Mount Sinai Hospital, University of Toronto, Toronto, Canada
*For correspondence: henrik. oster@uksh.de

†These authors contributed equally to this work

Present address: ${ }^{\ddagger}$ Department of Psychiatry, University of California, San Diego, San Diego, United States; ${ }^{\circledR}$ Center for Chronobiology, University of California, San Diego, San Diego, United States

Competing interests: The authors declare that no competing interests exist.

Funding: See page 14

Received: 23 December 2014

Accepted: 27 March 2015

Published: 30 March 2015

Reviewing editor: Louis Ptáček, University of California, San Francisco, United States

(c) Copyright Landgraf et al. This article is distributed under the terms of the Creative Commons Attribution License, which permits unrestricted use and redistribution provided that the original author and source are credited.
Abstract Circadian clocks coordinate 24-hr rhythms of behavior and physiology. In mammals, a master clock residing in the suprachiasmatic nucleus $(\mathrm{SCN})$ is reset by the light-dark cycle, while timed food intake is a potent synchronizer of peripheral clocks such as the liver. Alterations in food intake rhythms can uncouple peripheral clocks from the $\mathrm{SCN}$, resulting in internal desynchrony, which promotes obesity and metabolic disorders. Pancreas-derived hormones such as insulin and glucagon have been implicated in signaling mealtime to peripheral clocks. In this study, we identify a novel, more direct pathway of food-driven liver clock resetting involving oxyntomodulin (OXM). In mice, food intake stimulates OXM secretion from the gut, which resets liver transcription rhythms via induction of the core clock genes Per1 and 2. Inhibition of OXM signaling blocks food-mediated resetting of hepatocyte clocks. These data reveal a direct link between gastric filling with food and circadian rhythm phasing in metabolic tissues.

DOI: 10.7554/eLife.06253.001

\section{Introduction}

Extended night or rotating shift work is associated with an elevated risk for developing cancer, cardiovascular disease, immune deficiency, mood disorders, and metabolic alterations (Rosenberg and Doghramji, 2011; Herichova, 2013). One major factor believed to contribute to this adverse health impact of shift work is a disruption of endogenous circadian clocks by mistimed resetting stimuli, so called Zeitgebers, as a consequence of altered sleep/wake schedules. Most organisms have evolved internal timekeepers to anticipate the environmental changes brought about by the Earth's rotation around its axis. In mammals, these so called circadian clocks are based on ubiquitously expressed cellular interlocking transcriptional-translational feedback loops (TTLs) of clock genes/ proteins (Albrecht, 2012). In the core TTL, the transcriptional activators circadian locomotor output cycles kaput (CLOCK) and brain and muscle ARNT-like 1 (BMAL1; ARNTL) regulate the expression of two Cryptochrome (Cry1/2) and three Period (Per1-3) genes. Towards the end of the day, PER and CRY proteins translocate into the nucleus where they inhibit their own abundance via inhibition of CLOCK/BMAL1. Further accessory loops serve to stabilize this 24 -hr feedback rhythm and integrate the clock with cellular processes (Takahashi et alo, 2008). The clock machinery regulates physiology via orchestration of tissue-specific rhythmic expression of clock output genes (Yan et al., 2008).

The external light-dark cycle is the most prominent Zeitgeber of the central circadian pacemaker located in the suprachiasmatic nucleus (SCN) of the hypothalamus (Golombek and Rosenstein, 2010). The SCN receives light information from the retina and synchronizes peripheral clocks throughout the body via neuronal and hormonal pathways (Dibner et al., 2010). While the SCN itself is largely non-responsive to non-photic timing signals such as food intake, meal timing is an important 
eLife digest Humans and other animals have adapted their behavior and their biology to the daily cycle of light and dark. Groups of genes are reliably switched on or off at different times of the day, and act as internal, or 'circadian', clocks that help these organisms to stay on a 24-hour cycle. External signals also synchronize the body's internal clocks. For example, sunlight helps synchronize the master clock in the brain, while mealtimes and other cues help other organs keep time.

These internal clocks are often disrupted in people who work overnight or on rotating shifts. It is believed that when these individuals wake up or go to sleep at odd times it confuses their circadian clocks, which can be harmful to their health. People who work these unusual hours are at an increased risk of developing cancer, heart disease, obesity, and other disorders that involve problems with metabolism.

Eating at odd hours may also throw off the circadian clocks in the digestive system. This may explain why metabolic problems have been linked to working odd hours. Landgraf, Tsang et al. hypothesized that if the hormones produced after eating are released when a person would normally be sleeping, this may desynchronize the circadian clock in organs like the liver. Screening mice and tissue samples from mice for hormones that perturb circadian rhythms showed that a hormone called oxyntomodulin, which is released from the gut after eating, activated important circadian clock genes in mouse livers. The increases in clock gene activation were comparable to those seen in the brain in response to exposure to light.

Landgraf, Tsang et al. revealed that the clock-resetting effects of oxyntomodulin were the greatest when animals were exposed to it by eating, or by injections of the hormone, at times when the animals would normally be fasting. The experiments also showed that blocking oxyntomodulin prevented eating at unusual times from interfering with the liver's circadian clocks. The findings may suggest a way to help protect people who work overnight from the harmful health effects linked to perturbed circadian clocks.

DOI: 10.7554/eLife.06253.002

Zeitgeber for clocks in peripheral tissues (Stokkan et al., 2001). If food access is restricted to the normal rest phase of an organism, that is, the night for humans or daytime for nocturnal rodents, peripheral clocks become uncoupled from the SCN and adapt to the timing of food availability (Damiola et al., 2000). Shift workers often eat at times when their digestive timing system is poorly prepared for food (Lowden et al., 2010). Animal studies suggest that food intake during the normal rest phase promotes obesity (Arble et al., 2009; Hatori et al., 2012) and peripheral circadian uncoupling has been suggested to contribute to the development of metabolic disorders in night shift workers (Antunes et al., 2010; Barclay et al., 2012). Various other factors can regulate clock gene expression in peripheral tissues, including glucocorticoids and changes in body temperature or autonomic signaling (Dibner et al., 2010). The mechanisms of food-dependent peripheral clock resetting, however, remain poorly understood. Metabolic hormones such as insulin, ghrelin, and glucagon (GCG) have been shown to affect circadian rhythms associated with food restriction (LeSauter et alı, 2009; Tahara et alı, 2011; Chaves et al., 2014; Sun et al., 2015). While ghrelin appears to act primarily on the brain, insulin and GCG levels are mainly regulated via blood glucose. However, it was shown that carbohydrate intake alone has only a minor phase resetting capacity, while complex foods show much stronger effects (Hirao et al., 2009), indicating that other factors must be involved. Besides the pancreas, other organs-notably including the gastrointestinal tract itself-show acute hormonal responses to fasting or feeding (Stanley et al., 2005). This led us to hypothesize that postprandial, gut-derived signals may be implicated in food-driven resetting of peripheral clocks. In a screen using rhythmic liver slice cultures, we identified oxyntomodulin (OXM) as a potent resetting signal of liver circadian clocks. OXM is an anorexigenic incretin hormone produced in the gut by prohormone convertase 1/3-driven cleavage of the precursor preproglucagon (for review see Drucker, 2005). It modulates energy and glucose metabolism by acting on various tissues, including brain, liver, and pancreas (Baldissera et al., 1988; Gros et al., 1993). Since OXM secretion is dependent on food intake, we hypothesized that OXM may directly link food intake to hepatic transcriptional activity by resetting of the liver clock. 


\section{Results}

\section{OXM resets the circadian clock in organotypic liver slice cultures}

We screened a commercially available metabolic peptide library (Obesity Peptide Library, Phoenix Europe $\mathrm{GmbH}$; DE) for factors capable of resetting luciferase activity rhythms in organotypic liver slice cultures from Per2::LUC circadian reporter mice (Yoo et al., 2004). Interestingly, out of 200 peptides applied during the descending phase $\left(\sim 180^{\circ}\right.$, corresponding to the early morning) of the luciferase activity rhythm, only a few produced marked phase shifts, including three proglucagon-derived peptide (PGDP) hormones: exendin-4, OXM, and GCG (Figure 1-source data 1). Exendin-4 has been isolated from the salivary gland of the Gila monster, with no analogue in rodents or humans. To compare the effectiveness of mammalian PGDPs in liver clock resetting, we treated slices with increasing doses of OXM, GCG, and the three other commercially available PGDPs, glicentin-related pancreatic polypeptide (GRPP), glucagon-like peptide-1 (GLP-1), and glucagon-like peptide-2 (GLP-2) (Figure 1A). GLP-1, GLP-2, and GRPP (0.5-450 nM) had no significant resetting effects on PER2::LUC phase compared to PBS-treatment (Figure 1B-D). GCG resulted in phase delays of up to $3 \mathrm{hr}$, but only at relatively high concentrations (Figure 1E). In contrast, OXM reset PER2::LUC rhythms in liver slices at much lower doses, resulting in phase delays of up to $8 \mathrm{hr}$ at higher concentrations (Figure 1F). To act as a true Zeitgeber signal one would expect differential OXM effects depending on treatment time, that is, a circadian gating effect. We tested this by applying OXM at different phases of the PER2::LUC rhythm. To validate the setup, slices were treated at different PER2::LUC phases with 100 $\mu \mathrm{M}$ of the glucocorticoid analog dexamethasone (DEX), which was previously shown to reset hepatocyte clocks in vivo (Balsalobre et al., 2000). In a phase-dependent manner DEX treatment reset PER2::LUC activity rhythms in slices (Figure 1-figure supplement 1). Very similar to what had been observed after DEX treatment in animals (Balsalobre et al., 2000), application in the first quarter of the PER2::LUC activity rhythm (0-90') resulted in phase delays, while later treatments produced phase advances $\left(100-180^{\circ}\right.$ ) or had no marked effect (around $270^{\circ}$ ). Likewise, OXM effects were phase dependent. Delays were predominantly observed at $90-210^{\circ}$ of the PER2::LUC cycle with a maximum around $180^{\circ}$, while only modest phase shifts were seen at $270-360^{\circ}$ (Figure 1G). Though GCG also showed potential in resetting liver clock rhythms, OXM emerged as the most potent liver clock synchronizer from our screen. Moreover, contrary to GCG, OXM secretion is directly induced by food consumption in humans (Le Quellec et al., 1992), making it an attractive candidate for linking meal timing and clock function. Therefore, we focused on OXM for further analyses.

\section{OXM signals via the GCG receptor to activate Per gene expression in liver slices}

So far no OXM-specific receptor has been identified; however, OXM can bind to and activate both GCG and GLP-1 receptors (Jorgensen et al., 2007). Gcgr transcripts are strongly expressed in the murine liver (Sinclair et al., 2008). In contrast, the majority of previous studies failed to detect a fulllength Glp1r mRNA in murine hepatocytes (Campos et al., 1994; Dunphy et al., 1998; Panjwani et al., 2013). We performed RT-PCR analyses for all annotated coding Glp1r exons on cDNA preparations from wild-type mouse livers with pancreas as positive control. Glp1r transcripts were present in pancreas, but undetectable in liver samples (Figure 2-figure supplement 1), in line with the absence of significant liver clock resetting effects of GLP-1 (Figure 1B) and potent resetting of PER2::LUC rhythms by OXM treatment in slices from PER2::LUC x Glp1r ${ }^{-1-}$ mice (Figure 2A). On the other hand, blocking glucagon receptors (GCGR) signaling by co-treatment with 2-(4-Pyridyl)-5-(4chlorophenyl)-3-(5-bromo-2-propyloxyphenyl)pyrrol (Calbiochem Glucagon Receptor Inhibitor II; GRI-2) potently inhibited GCG- and OXM-induced clock resetting in Per2::LUC slices (Figure 2B).

GCGR is a G protein-coupled receptor that, via protein kinase A, leads to phosphorylation and activation of the transcription factor cyclic adenosine monophosphate (cAMP) response elementbinding protein (CREB) (Gonzalez and Montminy, 1989; Dalle et al., 2004). This pathway is reminiscent of the SCN, where nocturnal light exposure induces Per gene transcription via cAMP signaling and CREB activation downstream of the $\mathrm{N}$-methyl-D-aspartate receptor (Welsh et al., 2010). To investigate if OXM would impinge on the hepatic clock machinery in a similar way, we treated liver explants with OXM and performed chromatin immunoprecipitation (ChIP) analysis to measure CREB binding to cAMP response elements (CRE) in the Per1 gene promoter. 30 min after 


\section{A}

PROGLUCAGON

\begin{tabular}{|l|c|c|c|c|c|}
\hline GRPP & GCG & IP-1 & GLP-1 & IP-2 & GLP-2 \\
\hline & OXM \\
GLIC
\end{tabular}

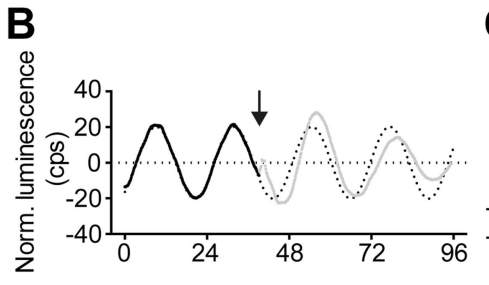

C
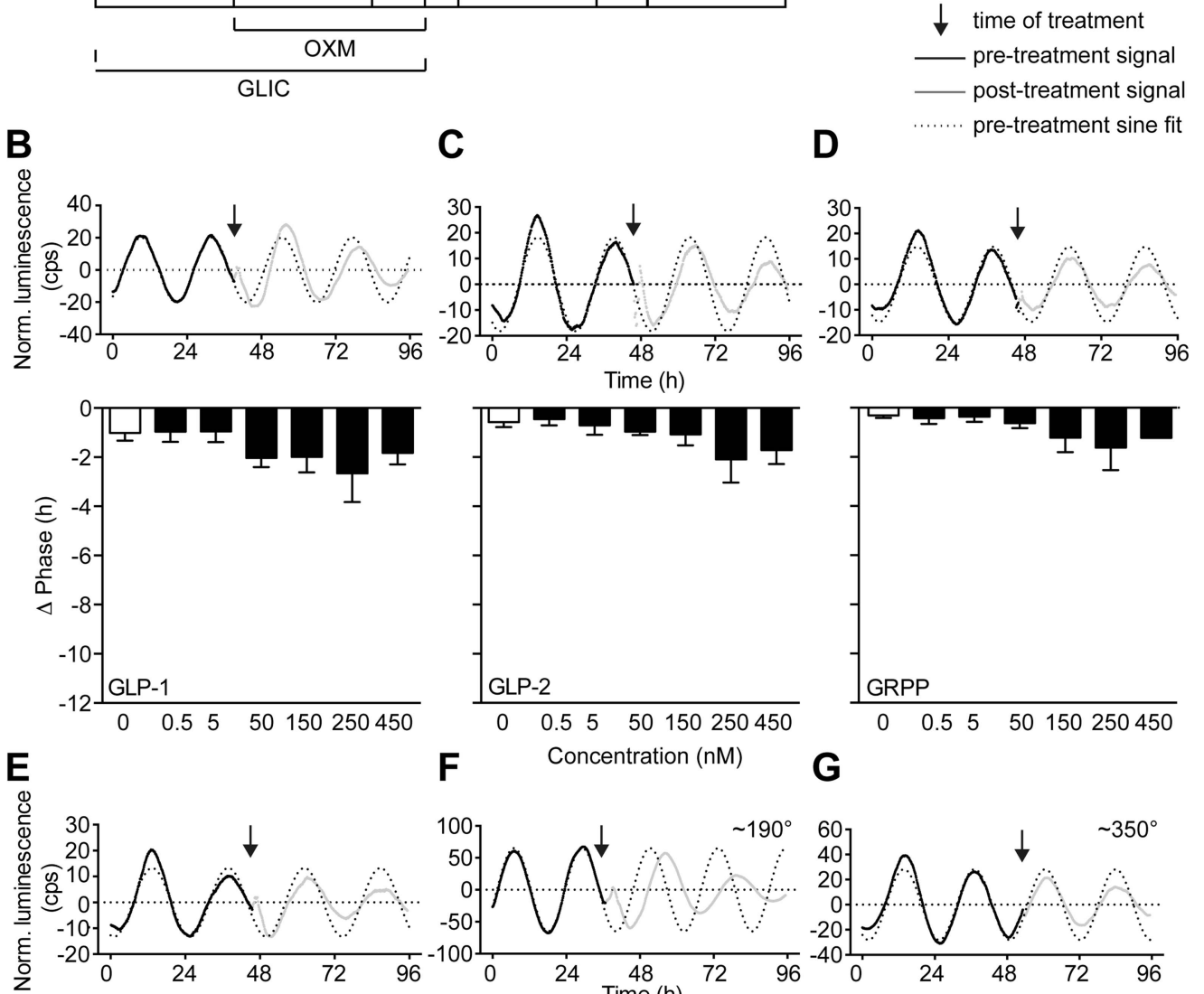

$F$

G
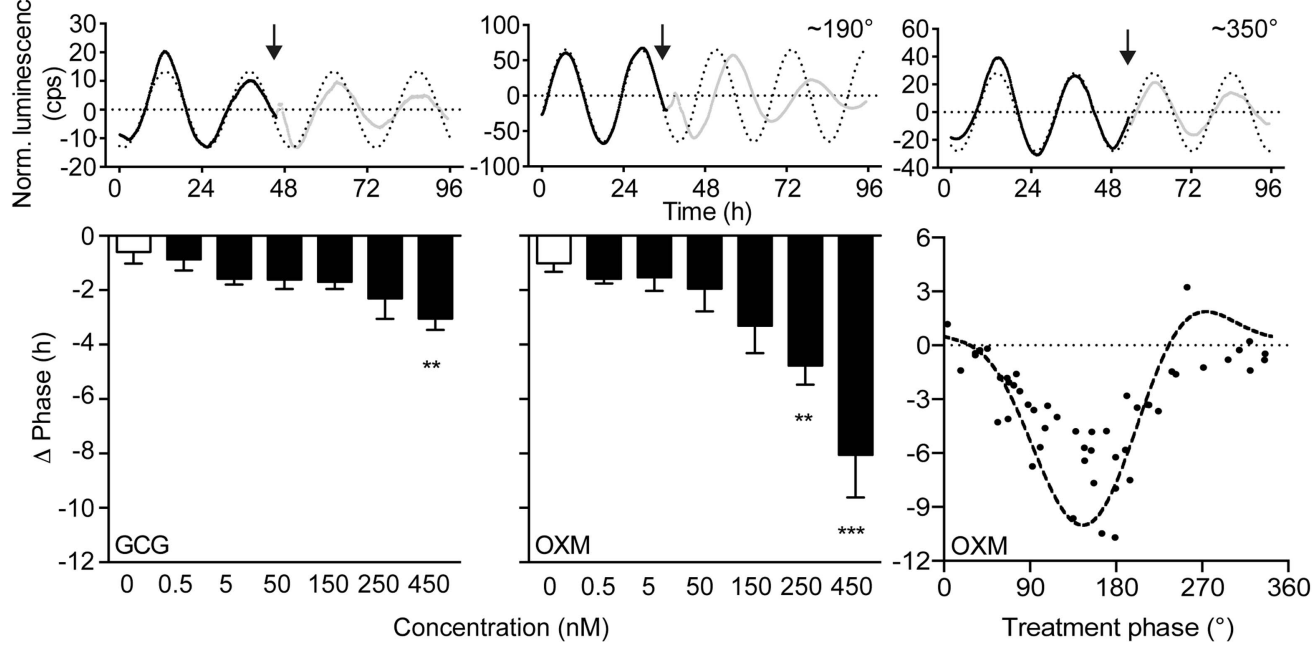

Figure 1. Oxyntomodulin (OXM) phase- and dose-dependently resets circadian clocks in liver slices. (A) Schematic sequence of the proglucagon-derived peptides (GRPP—glicentin-related pancreatic peptide; GLIC_glicentin; OXM—oxyntomodulin; GCG—glucagon; IP-1—intervening peptide-1; GLP-1—glucagon-like peptide-1; IP2-intervening peptide-2; GLP-2 - glucagon-like peptide-2). (B-F) Example luminescence traces and dosedependent responses for GLP-1 $(\mathbf{B} ; \mathbf{F}(6,28)=1.509)$, GLP-2 $(\mathbf{C} ; F(6,28)=1.530)$, GRPP $(\mathbf{D} ; F(6,28)=1.151)$, GCG (E; $F(6,28)=3.569)$, and OXM $(\mathbf{F} ; F(6,28)=8.790)$-induced phase resetting of PER2::LUC rhythms in liver slices treated at 180-200. Data are presented as mean \pm S.E.M. $(n=5)$. One-way ANOVA (F-values with degrees of freedom provided in brackets): ${ }^{\star} p<0.05 ;{ }^{* \star} p<0.01 ;{ }^{* \star} p<0.001$. Asterisks indicate significant differences relative to PBS treatment (white bars). (G) Phase response curve for OXM-induced phase resetting of PER2::LUC rhythms in liver slices. Circles: raw data of individual slices; dashed line: sine wave regression with harmonics.

DOI: 10.7554/eLife.06253.003

Figure 1. continued on next page 
Figure 1. Continued

The following source data and figure supplements are available for figure 1:

Source data 1. Table of effects of metabolic peptide treatment on PER2::LUC liver slice rhythms. DOI: 10.7554/eLife.06253.004

Figure supplement 1. Phase response curve for dexamethasone (DEX) treatment in Per2::LUC liver slice cultures. DOI: 10.7554/eLife.06253.005

OXM treatment, CREB binding was significantly increased at the Per1 CRE, but not at downstream sequences (Figure 2C). In addition, we analyzed clock gene expression in liver slices at different intervals after treatment with $\mathrm{OXM}$ at $180^{\circ}$. Per1 expression was transiently induced $60 \mathrm{~min}$ after addition of OXM to the medium, returning back to normal levels after 120 min (Figure 3A). Similarly, Per2 was induced by OXM after $60 \mathrm{~min}$, but mRNA levels remained high even after $120 \mathrm{~min}$ (Figure 3B). No significant effect was seen on Bmal1 expression at all time points (Figure 3C). In line with the absence of OXM-induced phase shifts at $360^{\circ}$ (Figure 1G), Per1/2 and Bmal1 mRNA levels were unaffected by OXM treatment at this phase (Figure 3D-F). Induction of Per1 and Per2 expression was preserved in Glp1 $r^{-/-}$slices, suggesting that hepatic OXM effects are independent of GLP-1R signaling (Figure 3G,H).

\section{OXM resets the liver clock in vivo}

To test if the effect of OXM on liver clock gene activity is preserved in vivo, we analyzed hepatic Per1/ 2 transcription after OXM treatment in wild-type mice. Analogous to what we observed in slices, robust Per1 and Per2 induction was observed after i.v. injection of OXM at Zeitgeber time (ZT) 3 (Figure 4A). When animals were treated with OXM at the opposite phase of the LD cycle (ZT15) a time when nocturnal mice usually eat and, thus, no food-mediated clock shifts would be expected-no induction of Per gene expression was observed (Figure 4B). Of note, in situ hybridization (ISH) showed no acute effect of OXM treatment on Per expression in the SCN at ZT3 (Figure 4C), indicating that OXM acts primarily on the liver clock and in line with the observed phase stability of the SCN clock under time-restricted feeding conditions (Damiola et al., 2000).

To assess OXM effects on liver clock phase, we next treated wild-type mice with either PBS or OXM at the beginning of their rest phase on the first day in constant darkness (DD) and under fasting conditions, thus excluding potential confounding effects of light exposure or food intake. Per2 and Dbp expression were determined from liver cDNA preparations at different time points using qPCR. We detected phase delays of Per2 and Dbp mRNA rhythms in livers of OXM-treated mice relative to those in PBS-injected control animals (Figure 4D), in line with the phase-delaying effects of OXM administration in slices at this time (Figure 1G). In addition, Per2, but not Dbp, rhythms appeared dampened after OXM injection.

Tissue clocks regulate local physiology via coordination of transcriptional programs. To test if OXM treatment would impinge on hepatic energy metabolism, we analyzed the expression of important metabolic transcripts after OXM treatment. Similar to what we observed for clock gene activity, transcript profiles of genes involved in liver carbohydrate metabolism were found either phase delayed (Foxo1 and Pdk4; Figure 5A,B) and/or dampened (Foxo1 and Pklr; Figure 5A,C). Of note Pepck, which was previously described as a clock output gene in liver (Lamia et al., 2008) was not rhythmic under these conditions, and OXM had no further effect on Pepck mRNA levels (Figure 5D). Expression levels of the glucose transporter Slc2a2 (Glut2) and the pyruvate transporter Slc16a7 were also dampened or phase-delayed, respectively (Figure 5E,F).

In summary, our data so far show that OXM treatment resets liver circadian mRNA rhythms in a phase- and dose-dependent manner, indicating that it may be involved in food-induced resetting of the liver circadian clock and metabolic machinery.

\section{Food intake-mediated OXM induction and liver clock resetting}

In humans, OXM levels in the blood rise in response to food intake (Le Quellec et al., 1992). To test whether this effect is conserved in mice, we determined diurnal plasma oxyntomodulin-like immunoreactivity (OLI) profiles in mice with ad libitum food access and in fasted animals. In fed mice, OLI levels were elevated during the active dark phase, while under fasting conditions OLI concentrations were constant and consistently low (Figure 6A). In line with this, non-rhythmic Gcg 


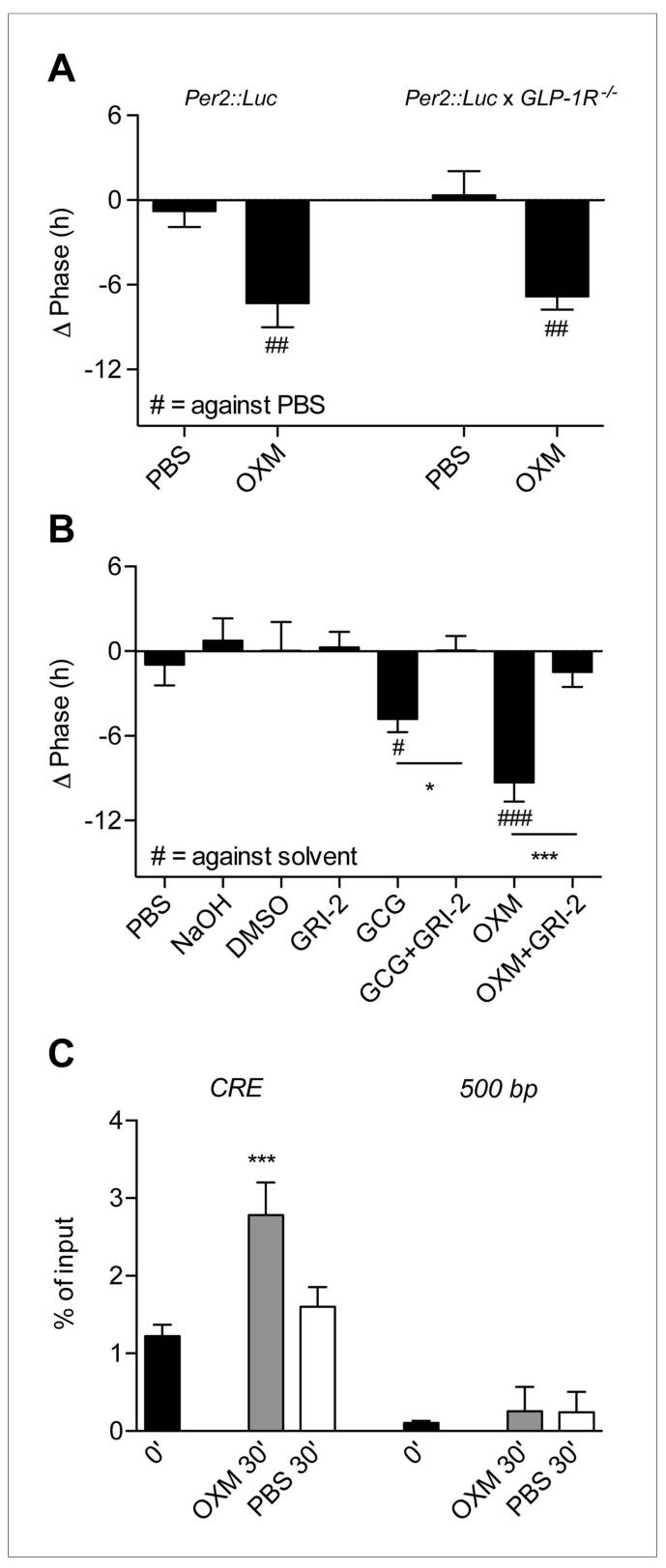

Figure 2. Glucagon (GCG) receptor regulates phase resetting effects of OXM and GCG in Per2::LUC liver slices. (A) OXM-induced phase shifts in Per2::LUC and Per2::LUC x Glp1r ${ }^{-1-}$ liver slices. Mann-Whitney test: \#\#p < 0.01 against solvent. (B) GCG and OXM-induced phase shifts in Per2::LUC slices are abolished by co-treatment with GRI-2. One-way ANOVA with Bonferroni post-test: $p<$ 0.05; \#\#\# $<0.001$ against solvent; ${ }^{\star} p<0.05 ;{ }^{* \star \star} p<0.001$. Data are presented as mean \pm S.E.M. $(n=8) ; F(7,56)=$ 7.314. (C) OXM treatment promotes binding of CREB to CRE elements at the Per1 gene promoter. One-way ANOVA with Bonferroni post-test: ${ }^{\star \star *} p<0.001$ against $0^{\prime}$. Data are presented as mean \pm S.E.M. $(n=5 ; F(5,24)=22.2)$. DOI: 10.7554/eLife.06253.006

The following figure supplement is available for figure 2 :

Figure supplement 1 . Absence of Glp1r transcripts in mouse liver.

DOI: 10.7554/eLife.06253.007
mRNA expression in the gut was observed under fasting conditions (data not shown). These data suggest a link between food intake and OXM secretion. To test this more directly, we used a 12-hr fasting-refeeding paradigm. Mean fasting OLI levels in the early morning (ZT1) were $\sim 3.5 \mathrm{ng} / \mathrm{ml}$, but showed high inter-individual variation (Figure 6B). Upon food intake, a rapid increase (relative to individual fasting levels) was observed after $20 \mathrm{~min}$. This effect persisted for more than $1 \mathrm{hr}$ before returning to baseline levels (Figure 6C). Of note, OLI induction after OXM injections were about twofold-threefold higher than what was observed after refeeding (Figure 6-figure supplement 1). To test if postprandial OLI induction is sufficient to affect liver clock gene expression, we analyzed Per1/2 mRNA levels in livers of wild-type mice after fasting-refeeding. Parallel to the rise in plasma OLI, we observed a transient postprandial increase of hepatic Per1 expression. Per2 expression showed a delayed, but a more persistent induction (Figure 6D). Food-mediated Per activation was partly inhibited by treatment with purified anti-OXM IgG to neutralize the effects of endogenous OXM in wild-type (Figure 6E) and in Glp1r ${ }^{-1-}$ mice (Figure 6F). Importantly, the effects of refeeding on insulin, GLP-1, and GCG plasma levels were not affected by treatment with anti-OXM IgG, suggesting that these peptides are not involved in the activation of foodinduced hepatic Per expression (Figure 6G-I).

We next tested if OXM signaling regulates foodmediated phase resetting of the liver clock using Per2::LUC slice preparations. Per2::LUC mice were starved overnight (ZT12-24/0) and treated with either IgG or anti-OXM antibodies prior to refeeding or extended starving. Animals were sacrificed at ZT4 and liver slices were prepared to determine luciferase rhythm phases. After a 12-hr fast, food intake caused a significant phase delay of PER2::LUC activity in liver slices and this effect was attenuated by OXM neutralization (Figure $6 J, K$ ). No effect on PER2::LUC phase was seen after antiOXM treatment alone (Figure 6K).

Together, these data suggest that elevated OXM levels in response to food intake affect hepatic clock gene expression. Neutralization of endogenous OXM signaling inhibits foodinduced clock gene induction and rhythm shifts, suggesting that OXM may act as a metabolic synchronizer of hepatic clocks.

\section{Discussion}

Food intake resets circadian clocks in peripheral tissues. In consequence, eating during the normal 


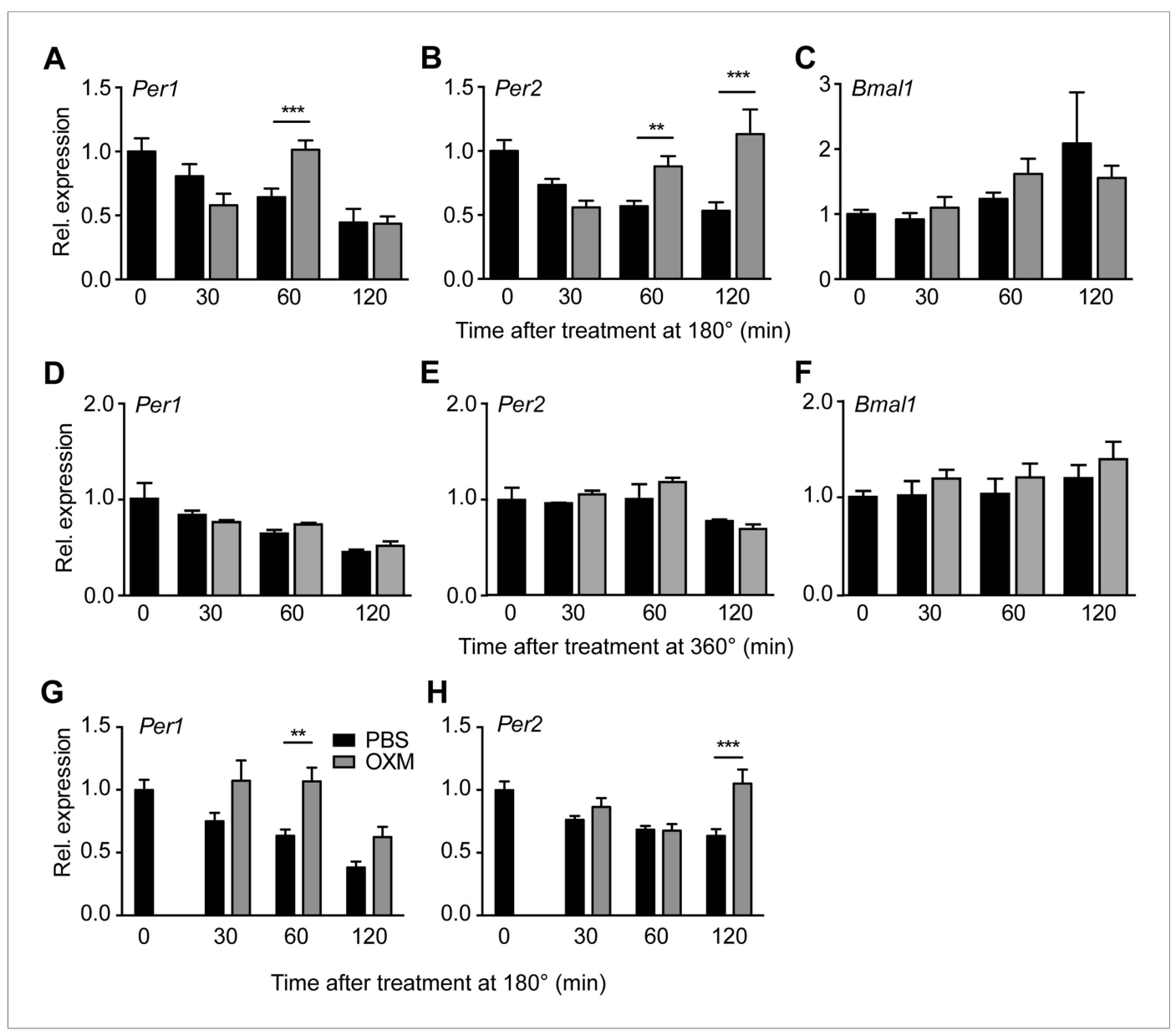

Figure 3. OXM treatment induces Per1/2 expression in organotypic liver slices. (A-C) WT liver slices were treated with OXM (grey) or vehicle (PBS; black) at $180^{\circ}$ and analyzed for clock gene expression of Per1 (A; factor treatment $F(1,40)=0.785$; time $F(3,40)=34.95$; interaction $F(3,40)=16.33)$, Per2 $(\mathbf{B}$; factor treatment $F(1,40)=24.02$; time $F(3,40)=29.4$; interaction $F(3,40)=38.38$ ), and Bmal1 $(\mathbf{C}$; factor treatment $F(1,40)=0.108$; time $F(3,40)=17.39$; interaction $F(3,40)=3.607$ ) by qPCR. (D-F) WT liver slices were treated with OXM (grey) or PBS (black) at $360^{\circ}$ and analyzed for expression of Per1 (D; factor treatment $F(1,40)=1.179$; time $F(3,40)=36.33$; interaction $F(3,40)=$ 1.349), Per2 $(\mathbf{E}$; factor treatment $F(1,40)=5.757$; time $F(3,40)=13.57$; interaction $F(3,40)=1.135)$, and Bmal1 $(\mathbf{F}$; factor treatment $F(1,40)=4.112$; time $F(3,40)=8.788$; interaction $F(3,40)=0.491)$ by qPCR. $(\mathbf{G}$ and $\mathbf{H})$ OXM-induced Per1/2 expression is retained in Glp1 $r^{-1-}$ liver slices. Per1: factor treatment $F(1,42)=8.48$; time $F(2,42)=10.95$; interaction $F(2,42)=0.525$. Per2: factor treatment $F(1,42)=10.5$; time $F(2,42)=3.662$; interaction $F(2,42)=5.845$. Glp $1 r^{-1-}$ liver slices were treated as described for WT above. Data are presented as mean \pm S.E.M. $(n=6$ for WT and 8 for Glp1 $\left.1 r^{-1-}\right)$. Two-way ANOVA with Bonferroni post-test: ${ }^{\star *} p<0.01 ;{ }^{* \star \star} p<0.001$.

DOI: 10.7554/eLife.06253.008

rest phase leads to a state of internal circadian desynchrony which has been suggested to underlie metabolic deregulation under shiftwork conditions (Vollmers et al., 2009; Salgado-Delgado et al., 2010; Barclay et al., 2012). Different mechanisms of food-related circadian synchronization have been proposed, including blood glucose-responsive peptide hormones (Dibner et al., 2010; Tahara et al., 2011; Chaves et al., 2014; Sun et al., 2015). However, food entrainment does not exclusively depend on glucose (Hirao et al., 2009), and the molecular mechanisms underlying food entrainment of peripheral tissues remain poorly characterized (Dibner et al., 2010). Here, we identified the incretin peptide OXM as a potential direct link between food intake in the gut and resetting of hepatic clock and metabolic gene transcription.

Using an organotypic slice culture system, we found that OXM-and, as was shown recently, GCG (Sun et al., 2015)—treatment can reset PER2::LUC circadian reporter activity rhythm in a dose- and phase-dependent manner (Figure 1). Consistently, peripheral OXM injections in mice reset clock gene 


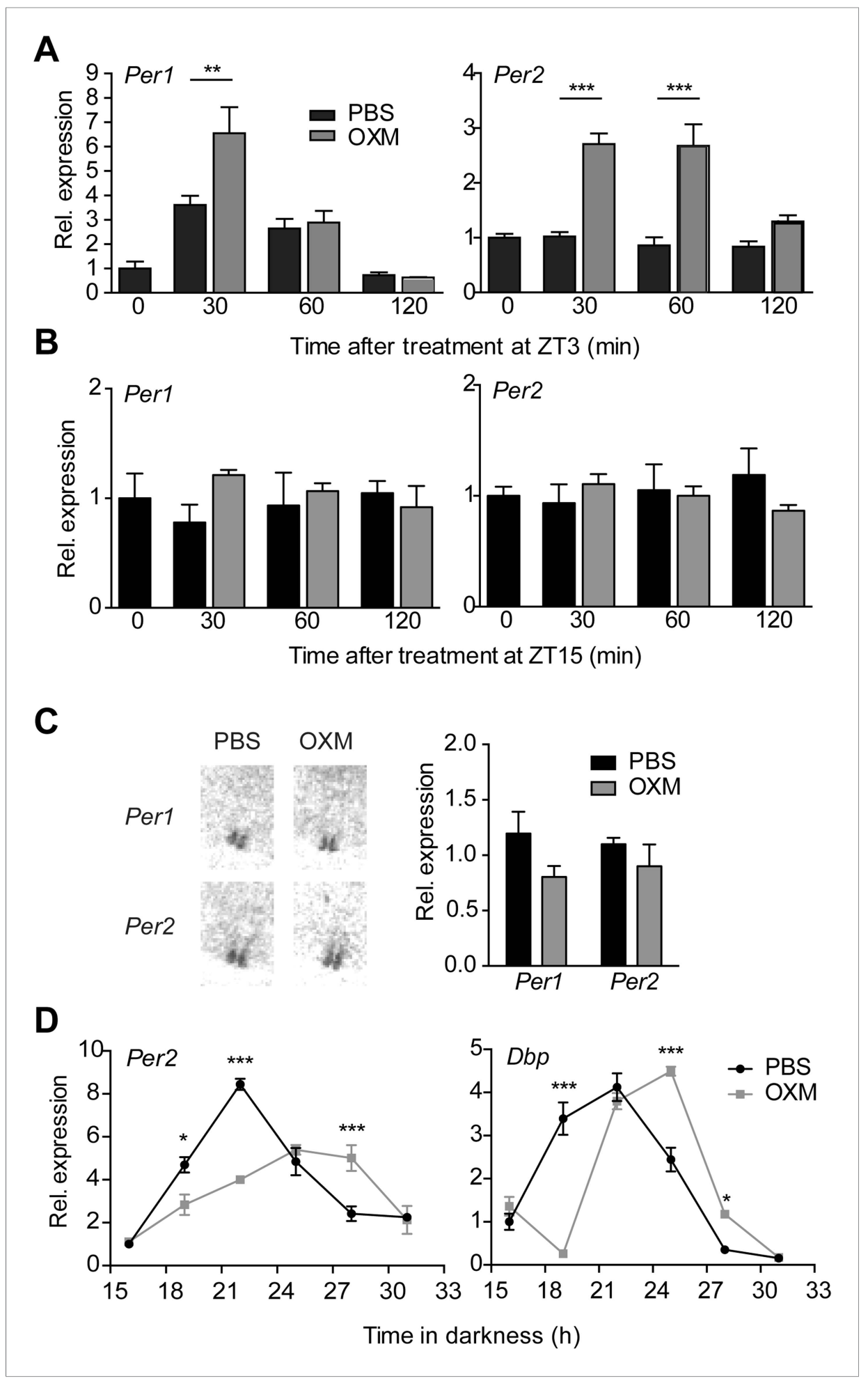

Figure 4. OXM treatment induces Per1/2 expression and resets the liver circadian clock in vivo. (A and B) Hepatic Per gene expression after OXM (grey) or vehicle (PBS; black) i.v. injection at ZT3 (A) and ZT15 (B). ZT3: Per1: factor treatment $F(1,24)=5.695$; time $F(2,24)=34.74$; interaction $F(2,24)=4.965$; Per2: factor treatment $F(1,24)=64.84$; time $F(2,24)=9.381$; interaction $F(2,24)=6.915$. ZT15: Per1: factor treatment $F(1,12)=1.096$; time $F(2,12)=0.005$; interaction $F(2,12)=1.367$; Per2: factor treatment $F(1,24)=0.255$; time $F(2,24)=0.001$; interaction $F(2,24)=1.172$. (C) Suprachiasmatic nucleus (SCN) signal after in situ hybridization (ISH) of brain sections with ${ }^{35} \mathrm{~S}$-labelled antisense probes for Per1/2 30 min after OXM/PBS treatment at ZT3 in the same animals used in (A). Left panel: representative Figure 4. continued on next page 
Figure 4. Continued

autoradiograph scans containing the SCN; right panel: quantification of the ISH. (D) Resetting of Per2 and Dbp rhythms in livers of wild-type mice after an i.p. injection of either OXM (grey) or vehicle (PBS; black) after 12-hr darkness; Per2: factor treatment $F(1,24)=5.531$; time $F(5,24)=46.37$; interaction $F(5,24)=18.71$. Dbp: factor treatment $F(1,24)=0.094$; time $F(5,24)=119.2$; interaction $F(5,24)=38.58$. All data are presented as mean \pm S.E.M. $(n=3-5)$. A, B, and D: two-way ANOVA with Bonferroni post-test: ${ }^{\star} p<0.05,{ }^{* \star} p<0.01 ;{ }^{* \star} p<0.001$; C: Mann-Whitney test.

DOI: 10.7554/eLife.06253.009

rhythms, as well as genes involved in hepatic carbohydrate regulation, thus impinging on hepatic energy metabolism (Figures 4, 5). In contrast, no significant effect of OXM treatment was observed at the level of the SCN pacemaker (Figure 4). In mice, food intake during day (i.e., the normal rest time) uncouples the liver clock from that in the SCN (Lamia et al., 2008), leading to a state of internal circadian desynchrony that is associated with elevated body weight and other metabolic impairments (Arble et al., 2009; Hatori et al., 2012). In the liver, around $10 \%$ of the transcriptome is under circadian regulation including many genes involved in energy metabolism (Akhtar et al., 2002; Miller et al., 2007). Genetic ablation of the liver clock abolishes the circadian rhythms of several glucose regulatory genes and results in a perturbed diurnal profile of blood glucose (Kornmann et al., 2001; Lamia et al., 2008).

Our experiments suggest that OXM signaling involves activation of GCGR (Figure 2). This is puzzling given that OXM displays a much greater capacity for clock gene induction in liver slices than GCG itself, despite having a lower binding affinity for GCGR (LeSauter et al., 2009). While we cannot conclusively answer this question at the moment, there are two scenarios that might explain our observations. First, GCGR may not be the only receptor involved in OXM-mediated clock resetting. While our data suggest that GLP1R does not play a role in this context (Figures 2, 3), an additional OXM receptor has been suggested (Baldissera et al., 1988; Sun et al., 2015), the activation of which may interact with GCGR downstream signaling. Second, an additional signal may be involved that acts synergistically with OXM to activate hepatic Per transcription. One such candidate could be insulin, which is similarly elevated after food intake and has previously been suggested to affect liver clock rhythms (Yamajuku et al., 2012; Chaves et al., 2014; Sun et al., 2015). In response to food intake OXM levels go up, while GCG plasma concentrations are reduced (Figure 6), suggesting that postprandial GCGR activation does not depend on GCG itself. Interestingly, GCG/GCGR signaling has recently been implicated in the regulation of hepatic Bmal1 expression in response to prolonged starvation (Sun et al., 2015). Sun et al. show that fasting-induced GCG signaling activates Bmal1 transcription via CREB/CRTC2 during the night. As we did not observe any acute effects of OXM treatment on Bmal1 transcription (Figure 3), these data suggest that GCGR signaling may have different clock targets depending on the time of activation. GCGR activation is known to induce protein kinase A-mediated nuclear translocation and DNA-binding of phosphorylated CREB on target genes (Gonzalez and Montminy, 1989; Dalle et al., 2004). In line with this, we show OXM-induced binding of CREB to Per1 promoter CRE motifs and induction of Per clock gene expression (Figures 2-4). Similar phase-dependencies were observed for Per induction as were seen for clock shifting (compare Figures 1, 3, 4). For various tissues_including the SCN_it has been shown that resetting of the circadian clock involves acute up-regulation of Per1 and Per2 (Dunlap, 1999; Lowrey and Takahashi, 2004). Per induction was observed after DEX treatment or serum shock in fibroblast and hepatoma cells (Balsalobre et al., 1998, 2000). Light pulses given during the dark phase induce CREB activation and Per expression in the SCN (Albrecht et al., 1997; Yan and Silver, 2002). In line with this, we showed Per1 and Per2 induction after OXM treatment. Of note, Per induction in liver slices was weaker than in vivo, suggesting that additional mechanisms may amplify OXM effects in intact animals. Along the same line, OXM-mediated Per induction in vivo appeared to be slightly faster than in slices (Figures 3, 4). Importantly, peripheral treatment with OXM also altered the diurnal expression rhythms of genes involved in regulating liver carbohydrate metabolism which may underline the metabolic effects of daytime feeding in rodents (Figure 5). Similar to the OXM effects on clock gene expression (Figures 3, 4), refeeding acutely induces hepatic Per1/2 expression, which has been proposed as an integral part of the food-driven clock-resetting mechanism (Oike et al., 2011; Tahara et al., 2011). In our study, we demonstrated that food intake after overnight fasting stimulated OXM 


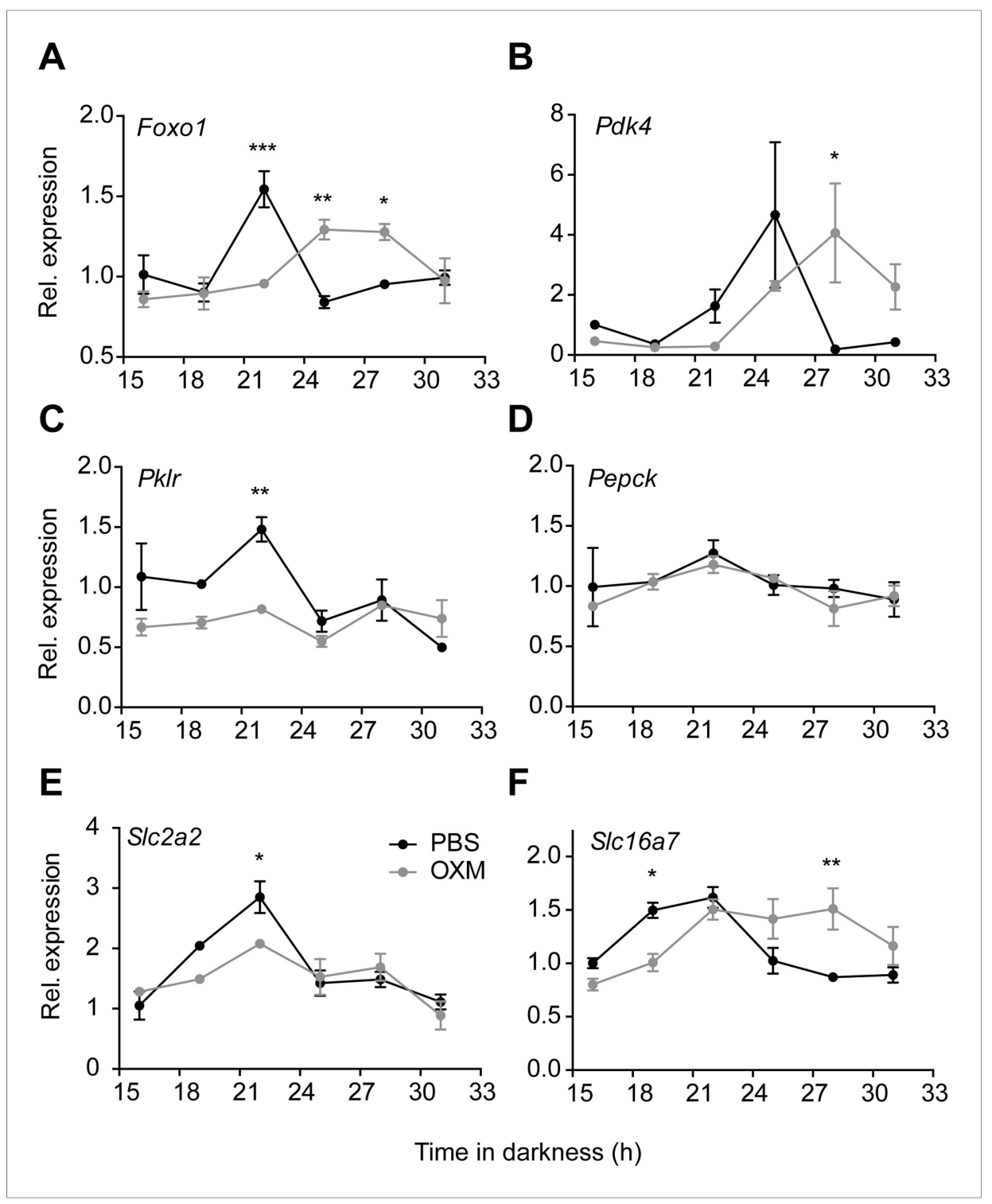

Figure 5. OXM treatment modulates diurnal expression profile of hepatic genes involved in liver carbohydrate metabolism. (A-F) Relative gene expression of Foxo1 (A; factor treatment $F(1,24)=0.001$, time $F(5,24)=5.547$, interaction $F(5,24)=11.13)$, Pdk4 $(\mathbf{B}$; factor treatment $F(1,24)=0.197$, time $F(5,24)=3.35$, interaction $F(5,24)=$ 3.247), Pklr (C; factor treatment $F(1,24)=11.63$, time $F(5,24)=5.61$, interaction $F(5,24)=3.61)$, Pepck $(\mathbf{D}$; factor treatment $F(1,24)=0.574$, time $F(5,24)=2.043$, interaction $F(5,24)=0.299)$, the glucose transporter Slc2a2 $\left(\mathbf{E}_{\text {; }}\right.$ factor treatment $F(1,24)=2.582$, time $F(5,24)=15.98$, interaction $F(5,24)=2.642$ ) and the pyruvate transporter Slc16a7 ( $F$; factor treatment $F(1,24)=1.539$, time $F(5,24)=7.472$, interaction $F(5,24)=6.586$ ) after i.p. administration of either OXM (grey) or vehicle (PBS; black) after $12 \mathrm{hr}$ in darkness. Data are presented as mean \pm S.E.M. $(n=4)$. Two-way ANOVA with Bonferroni post-test: ${ }^{\star} p<0.05,{ }^{\star \star} p<0.01 ;{ }^{\star \star *} p<0.001$.

DOI: 10.7554/eLife.06253.010

secretion and led to hepatic up-regulation of Per expression, which was blocked by OXM neutralization in the circulation. Accordingly, liver slice cultures from re-fed Per2::LUC reporter mice showed food-dependent phase delays and these effects were attenuated by OXM neutralization (Figure 6). OXM neutralization does not completely inhibit the effects of refeeding on Per induction and liver clock resetting. This might be due to incomplete neutralization of OXM or the contribution of other food-induced factors as discussed above. Of note, while our data do not provide evidence for a direct involvement of GLP-1(R), it has been suggested that postprandial GLP-1 signaling may indirectly affect liver clock phase (Panjwani et al., 2013). 


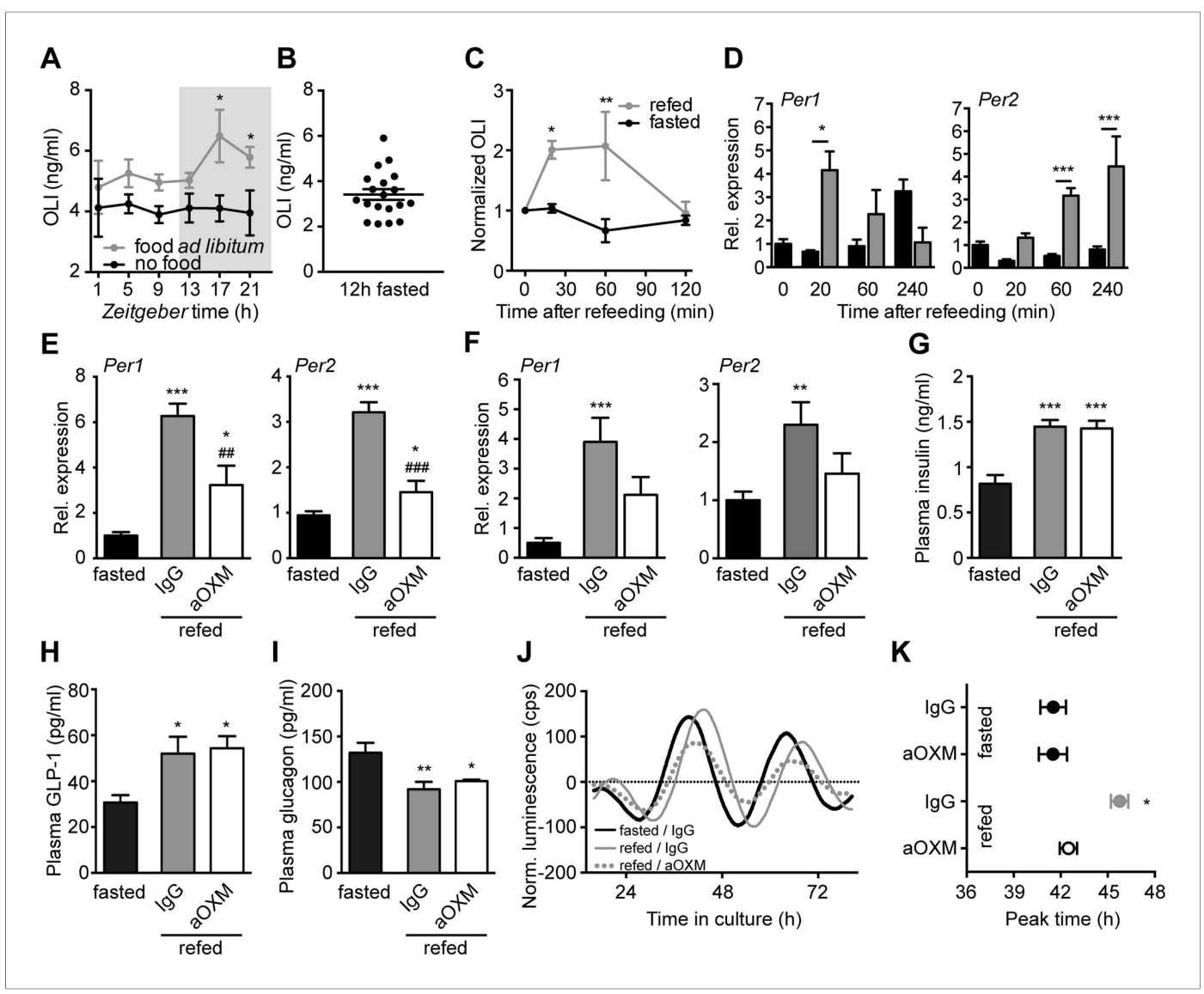

Figure 6. Endogenous OXM signaling regulates food intake-mediated resetting of the liver circadian clock. (A) Plasma oxyntomodulin-like immunoreactivity (OLI) diurnal profiles under ad libitum food and fasting conditions. Data are presented as mean \pm S.E.M $(n=6)$; factor time $F(5,60)=0.628$, feeding condition $F(1,60)=15.37$, interaction $F(5,60)=0.638$. Grey shading indicates the dark phase. (B) OLI levels show high individual variations in mice after $12 \mathrm{hr}$ of food deprivation (ZT13-1). (C) Plasma OLI (normalized to individual fasting levels) after refeeding (grey line) or under continuous starving (black line); factor time $F(3,21)=3.544$, feeding condition $F(1,21)=15.82$, interaction $F(3,21)=4.717$. (D) Liver Per1/2 induction following fasting-refeeding determined by qPCR; Per1: factor time $F(3,25)=0.454$, feeding condition $F(1,25)=1.376$, interaction $F(3,25)=4.453$; Per2: factor time $F(3,25)=6.938$, feeding condition $F(1,25)=38.48$, interaction $F(3,25)=3.767$. (E) WT and $(F) G / p 1 r^{-1-}$ liver Per1/2 expression after fasting-refeeding with control lgG injection (grey) or OXM immuno-neutralization by anti-OXM IgG (aOXM) injection at ZT0; WT: Per1: F(2, 12) = 71.76, Per2: F(2, 12) =47.41; Glp1r ${ }^{-1-}$ : Per1 F(2, 12) =11.51, Per2: F(2, 12) = 5.585. (G-I) Treatment with anti-OXM IgG does not affect postprandial regulation of insulin, GLP-1, and GCG. Plasma levels of insulin $(\mathbf{G} ; F(2,12)=17.44)$, $G L P-1(\mathbf{H} ; F(2,12)=5.563)$, and $G C G(\mathbf{I} ; F(2,12)=7.128)$ after fasting-refeeding with control IgG injection (grey) or OXM immuno-neutralization by anti-OXM IgG (aOXM) treatment at ZTO. One(E-I) or two-way ANOVA (A, C, D) with Bonferroni post-test: ${ }^{\star} p<0.05 ;{ }^{\star \star} p<0.01$; ${ }^{\star \star \star} p<0.001$ against fasted; $\# \# p<0.01$; \#\#\# < 0.001 against IgG. Data are presented as mean \pm S.E.M $(n=5)$. (J and K) Liver PER2::LUC rhythms after fasting-refeeding with control IgG or $\alpha O X M$ administration. (J) Representative luminescence traces. (K) Comparison of phases (second peak in culture) after refeeding and/or anti-OXM treatment (Data are presented as mean \pm S.E.M ( $n=4$ mice per condition, an average of 3 slice preparations of each mouse were used); two-way ANOVA with Bonferroni post-test: ${ }^{*} p<0.05$ against fasted; factor treatment $F(1,12)=5.127$, feeding condition $F(1,12)=13.02$, interaction $F(1,12)=5.044)$.

DOI: 10.7554/eLife.06253.011

The following source data and figure supplements are available for figure 6:

Source data 1. Primer sequences for PCR reactions.

DOI: 10.7554/eLife.06253.012

Figure supplement 1. Time course of OLI plasma levels after OXM injection. DOI: 10.7554/eLife.06253.013 
Our data indicate an involvement of OXM signaling in food-driven resetting of the liver circadian clock. Similar to what was previously reported in humans (Mayo et al., 2003), we detected elevated OLI levels in fed as compared to starved mice and in response to acute food intake (Figure 6). The diurnal variability of OLI under ad libitum feeding conditions was moderate, but fasting resulted in an approximate $20 \%$ reduction of diurnal OLI secretion. Together this indicates that small meals as consumed during the inactive phase affect OXM secretion, but that substantial food intake is necessary to acutely increase OXM secretion to an extent sufficient to affect the liver clock. Of note, interpretation of plasma OXM blood levels is difficult as many OXM assays exhibit some degree of cross-reactivity with GCG and the closely related glicentin. Glicentin differs from OXM only by a 32-amino acid N-terminal extension (IP-1; Figure 1) and is released from the gut after food intake at approximately similar levels (Blache et al., 1988; Tang-Christensen et al., 2001), thus we cannot exclude the possibility that glicentin may exhibit actions that overlap with those described for OXM.

In summary, we show that food intake induces OLI blood levels and hepatic clock resetting in mice. OXM treatment mimics food-mediated clock resetting in slices and in vivo in a time of day-dependent manner. Food is a major regulator of hepatic transcriptome rhythms (Vollmers et al., 2009). A role for metabolic hormones, for example, insulin, GCG, or glucocorticoids, in the regulation of peripheral clocks has been suggested (Balsalobre et al., 2000; Tahara et al., 2011; Sun et al., 2015). However, the mechanisms of food-related synchronization of peripheral clocks and their uncoupling from the SCN under time-restricted food intake rhythms are still poorly understood. Our data suggest that OXM-most likely in concert with other gut- and pancreas-derived hunger- or satiety-signaling peptides-is involved in the detrimental effects of mistimed food intake on metabolic homeostasis (Arble et al., 2009; Albrecht, 2012; Hatori et al., 2012). While interfering with insulin signaling may be clinically problematic because of its potential deleterious effects on glycemia, chronotherapeutic targeting of peripheral incretin signaling may provide an alternative therapeutic strategy against metabolic disorders arising from circadian strain as observed in shift workers or during jetlag.

\section{Materials and methods}

\section{Animal strains}

All animal experiments were ethically assessed and licensed by the Office of Consumer Protection and Food Safety of the State of Lower Saxony and in accordance with the German Law of Animal Welfare (license nos. V312-7224.122-4 and 33.12-42502-04-12/0893). For all experiments adult wild-type mice male C57BL/6J (8-24 weeks old) were used. If not stated otherwise, mice were provided with food and water ad libitum. To investigate the effect of OXM on gene expression by qPCR or ISH, mice were peripherally treated with OXM (i.v. $4 \mu \mathrm{g}$ /mouse; i.p. $25 \mu \mathrm{g} / \mathrm{mouse}$ ) or vehicle (PBS) at ZT3, ZT15, or 12 hr after light-off. For luminescence measurements adult heterozygous males Per2::LUC (Yoo et al., 2004) and Per2::LUC x Glp1r ${ }^{-1-}$ were used. Glp1r $r^{-1-}$ mice were maintained on a C57B/6J background (Scrocchi et al., 1996). All mice were exposed to a 12-hr: 12-hr light-dark cycle with 100 lux in the light phase (LD12:12). Animals were sacrificed at indicated time points by cervical dislocation. Animals euthanized during the dark phase were handled under red light and eyes were removed before dissection. All tissue samples were collected and immediately snap-frozen on dry ice or liquid nitrogen. For long-term storage tissues were kept at $-80^{\circ} \mathrm{C}$.

\section{Refeeding experiments}

In order to test Per induction and phase shifts after refeeding, mice were fasted for one night (13 hr, or from ZT12_ZT1) and either food deprived until decapitation or refed at ZT1. The refed mice received either anti-OXM rabbit lgG (i.p. $50 \mu \mathrm{g}$ /mouse; T-4800; Bachem, Bubendorf, Switzerland) or control lgG from unimmunized rabbit serum (15006; Sigma-Aldrich, Seelze, Germany) at ZT1 when food was returned.

\section{Liver slice cultures and peptide treatments}

Luminescence was measured from cultured liver slices of heterozygous Per2::LUC mice as described previously (Yoo et al., 2004) modified to include the use of culture plate inserts (Millipore, Billerica, MA). Briefly, the median lobe of the liver was isolated and $300-\mu \mathrm{m}$ slices were prepared using a vibratome (Campden Instruments, Loughborough, UK). The slices were immediately placed onto a culture plate insert in 35-mm petri dishes filled with 1-ml culture medium (D-MEM with high glucose, 
w/o L-glutamine and phenol red; Life Technologies, Darmstadt, Germany) supplemented with 3 mM sodium carbonate (Sigma-Aldrich), $10 \mathrm{mM}$ HEPES buffer, $2 \mathrm{mM}$ L-glutamine, 2\% B-27 supplement, $25 \mathrm{U} / \mathrm{ml}$ penicillin/streptomycin and $0.1 \mathrm{mM}$ D-luciferin (all Life Technologies). Luminescence was measured in a luminometer (Actimetrics, Evanston, IL) at $32.5^{\circ} \mathrm{C}$ with $5 \% \mathrm{CO}_{2}$. Analyses were performed using the LumiCycle analysis (Actimetrics) and Prism software packages (GraphPad, La Jolla, CA). PER2:: LUC activity in slices closely follows a sine wave shape. The intersection of the ascending cross-section of the sine wave with the $x$-axis was defined as $0^{\circ} / 360^{\circ}$, the peak as $90^{\circ}$. Degrees at the time point of treatment were calculated as follows: $T_{p}\left[{ }^{\circ}\right]=\left(\left(T_{p}[\mathrm{hsm}]-P_{b t}[\mathrm{hsm}]\right): P_{b t}\right) \times 360+90$ with $T_{p}=$ treatment phase; ${ }^{\circ}=$ degree; $h s m=$ hours after start of measurement; $P_{b t}=$ peak before treatment. If the result was $>360^{\circ}$, the value was subtracted by 360 . Raw data were baseline subtracted with running averages of $24 \mathrm{hr}$. Peaks were defined as middle time point between two troughs of the sine wave. Period was determined as the time between peaks averaged over 2-3 consecutive cycles. For the duration of treatment, samples were maintained at $32.5^{\circ} \mathrm{C}$ to avoid resetting of clock gene expression rhythms due to temperature changes. Phase shifts were determined by comparing extrapolated peak times from sine wave fits before and after treatment. Unless otherwise stated, peptides used for experiments were dissolved in culture medium and administered at a final concentration of $450 \mathrm{pM}$.

\section{Quantitative real-time (qPCR)}

Quantitative real-time PCR (qPCR) was performed with a CFX96 thermocycler system (Bio-Rad, Munich, Germany) with iQ-SYBR Green SuperMix (Bio-Rad). Relative quantification of expression levels by a modified $\Delta \Delta C T$ calculation was performed as described (Pfaffl, 2001). B-Actin was used as a reference gene. Statistical analyses were performed using GraphPad Prism software. Circadian profiles of clock gene expression were normalized against the average values over all time points. Induction analyses were normalized against untreated conditions (0 min). PCR primer sequences are listed in Figure 6-source data 1.

\section{ISH}

The Per1 probe corresponds to nucleotides 1 to 619 (GenBank accession number AF022992) and Per2 corresponds to nucleotides 229 to 768 of GenBank AF036893. PCR products had been cloned into pCR II TOPO vector using TOPO TA Cloning Kit (Life Technologies) (Oster et al., 2002). Linearization of vectors for in vitro transcription was done with EcoRI. ${ }^{35} \mathrm{~S}-U T P$ (PerkinElmer, Waltham, MA) labeled RNA probes were prepared using RNA Transcription Kit (Maxi Script Labeling Kit, Life Technologies) with T7 or T3 RNA polymerases according to the manufacturer's protocol. $10-\mu \mathrm{m}$ cryosections were cut using a Leica CM3050 cryostat. Cryosections were fixed in 4\% paraformaldehyde, acetylated in acetic anhydride and dehydrated with ethanol. Hybridization was performed over night at $55-58^{\circ} \mathrm{C}$. Autoradiographs were analyzed by densitometry (Bio-Rad GS-800) using QuantityOne software (BioRad). Three sections per brain were used and background values were calculated from adjacent tissue areas on the same slide for each section. Measurements from different animals/experiments were compared for statistical analysis using GraphPad Prism (GraphPad).

\section{ChIP}

Liver slices were homogenized and immediately cross linked with $1 \%$ formaldehyde. Chromatin was sonicated for 15-s on/20-s off cycles for 22 min using a Bioruptor sonicator (Diagenode, Denville, NJ). Samples were incubated overnight at $4^{\circ} \mathrm{C}$ with CREB antibody (ab31387, Abcam, Cambridge, UK). After clearing, samples were incubated with A/G agarose beads (Thermo Scientific, Braunschweig, Germany) for $1 \mathrm{hr}$ at $4^{\circ} \mathrm{C}$ followed by repetitive washings. After boiling for $10 \mathrm{~min}$ in $10 \%$ Chelex (BioRad) with Proteinase K (150 mg/ml), samples were spun down and DNA-containing supernatant was collected for PCR. qPCR was performed as described above, and values were normalized to percentage of input. Primer sequences were: 5'-CAGCTGCCTCGCCCCGCCTC-3'/5'-CCCAAGCAGCCATTGCTCGC-3' (Per1 CRE) and 5'-CCCCGCAGTCCTACGGTGCTG-3'/5'-AAGCCCCCAAACAACTGAAGGT-3' (500 bp downstream sequence).

\section{Hormone measurements}

Blood collection for radioimmunoassay (RIA) was performed at ZT1 after $12 \mathrm{hr}$ fast. Mice were allowed to recover for 3 days, then fasted again followed by treatments (with or without refeeding). Blood was collected from the tail vein at $0 \mathrm{~min}, 20 \mathrm{~min}, 60 \mathrm{~min}$, and $120 \mathrm{~min}$ after treatment. Plasma concentrations of OLI were determined by RIA (Phoenix Pharmaceutics, Karlsruhe, DE) according to 
manufacturer's protocol modified to use a 50\% reaction volume. GLP-1 (EZGLP-1T-36K, Millipore, Darmstadt, Germany), insulin (Catalog\# 90080, CrystalChem, Downers Grove, IL), and GCG plasma levels (EZGLU-30K, Millipore) were determined by ELISA according to the manufacturers' protocols.

\section{Statistics}

Data were analyzed with GraphPad Prism (GraphPad). Mann-Whitney tests were used for simple comparisons. For dose responses one-way ANOVAs and for two-factor comparisons two-way ANOVAs with Bonferroni post-tests were used. A p-value of less than 0.05 was considered significant.

\section{Acknowledgements}

The authors thank Bernard Thorens, University of Lausanne for indirectly providing Glp1r ${ }^{-1-}$ mice, Nadine Naujokat and Christin Helbig for technical assistance, and David K Welsh, UC San Diego, and Gregor Eichele, Max Planck Institute for Biophysical Chemistry Göttingen, for critical comments on the manuscript.

\section{Additional information}

Funding

\begin{tabular}{|c|c|c|}
\hline Funder & Grant reference & Author \\
\hline Volkswagen Foundation & Lichtenberg Fellowship & Henrik Oster \\
\hline $\begin{array}{l}\text { Deutsche } \\
\text { Forschungsgemeinschaft (DFG) }\end{array}$ & TR-SFB134 & Henrik Oster \\
\hline $\begin{array}{l}\text { Canada Research Chairs (Chaires } \\
\text { de recherche du Canada) }\end{array}$ & & Daniel J Drucker \\
\hline $\begin{array}{l}\text { Canadian Institutes of Health } \\
\text { Research (Instituts de recherche } \\
\text { en santé du Canada) }\end{array}$ & 93749 & Daniel J Drucker \\
\hline Novo Nordisk & Chair of Incretin Biology & Daniel J Drucker \\
\hline
\end{tabular}

The funders had no role in study design, data collection and interpretation, or the decision to submit the work for publication.

Author contributions

$\mathrm{DL}, \mathrm{AHT}$, Acquisition of data, Analysis and interpretation of data, Drafting or revising the article; $\mathrm{AL}$, CEK, JLB, Acquisition of data, Analysis and interpretation of data; DJD, Drafting or revising the article, Contributed unpublished essential data or reagents; $\mathrm{HO}$, Conception and design, Acquisition of data, Analysis and interpretation of data, Drafting or revising the article

Ethics

Animal experimentation: This study was performed in strict accordance with the German law for animal welfare (TierschGes). All animals were handled according to approved institutional animal care and use committee protocols of the Max Planck Institutes Göttingen and the University of Lübeck. The protocol was approved by the ethical committees of the Niedersächsisches Amt für Verbraucherschutz und Lebensmittelsicherheit (LAVES) and the Ministerium für Energiewende, Landwirtschaft, Umwelt und ländliche Räume (MELUR; license numbers V312-7224.122-4 and 33.1242502-04-12/0893). Every effort was made to minimize suffering.

\section{References}

Akhtar RA, Reddy AB, Maywood ES, Clayton JD, King VM, Smith AG, Gant TW, Hastings MH, Kyriacou CP. 2002. Circadian cycling of the mouse liver transcriptome, as revealed by cDNA microarray, is driven by the suprachiasmatic nucleus. Current Biology 12:540-550. doi: 10.1016/S0960-9822(02)00759-5.

Albrecht U. 2012. Timing to perfection: the biology of central and peripheral circadian clocks. Neuron 74:246-260. doi: 10.1016/j.neuron.2012.04.006.

Albrecht U, Sun ZS, Eichele G, Lee CC. 1997. A differential response of two putative mammalian circadian regulators, mper1 and mper2, to light. Cell 91:1055-1064. doi: 10.1016/S0092-8674(00)80495-X.

Antunes LC, Levandovski R, Dantas G, Caumo W, Hidalgo MP. 2010. Obesity and shift work: chronobiological aspects. Nutrition Research Reviews 23:155-168. doi: 10.1017/S0954422410000016. 
Arble DM, Bass J, Laposky AD, Vitaterna MH, Turek FW. 2009. Circadian timing of food intake contributes to weight gain. Obesity 17:2100-2102. doi: 10.1038/oby.2009.264.

Baldissera FG, Holst JJ, Knuhtsen S, Hilsted L, Nielsen OV. 1988. Oxyntomodulin (glicentin-(33-69)): pharmacokinetics, binding to liver cell membranes, effects on isolated perfused pig pancreas, and secretion from isolated perfused lower small intestine of pigs. Regulatory Peptides 21:151-166. doi: 10.1016/0167-0115(88) 90099-7.

Balsalobre A, Brown SA, Marcacci L, Tronche F, Kellendonk C, Reichardt HM, Schütz G, Schibler U. 2000. Resetting of circadian time in peripheral tissues by glucocorticoid signaling. Science 289:2344-2347. doi: 10.1126/science. 289.5488.2344.

Balsalobre A, Damiola F, Schibler U. 1998. A serum shock induces circadian gene expression in mammalian tissue culture cells. Cell 93:929-937. doi: 10.1016/S0092-8674(00)81199-X.

Barclay JL, Husse J, Bode B, Naujokat N, Meyer-Kovac J, Schmid SM, Lehnert H, Oster H. 2012. Circadian desynchrony promotes metabolic disruption in a mouse model of shiftwork. PLOS ONE 7:e37150. doi: 10.1371/ journal.pone.0037150.

Blache P, Kervran A, Bataille D. 1988. Oxyntomodulin and glicentin: brain-gut peptides in the rat. Endocrinology 123:2782-2787. doi: 10.1210/endo-123-6-2782.

Campos RV, Lee YC, Drucker DJ. 1994. Divergent tissue-specific and developmental expression of receptors for glucagon and glucagon-like peptide-1 in the mouse. Endocrinology 134:2156-2164. doi: 10.1210/endo.134.5.8156917.

Chaves I, van der Horst GT, Schellevis R, Nijman RM, Koerkamp MG, Holstege FC, Smidt MP, Hoekman MF. 2014. Insulin-FOXO3 signaling modulates circadian rhythms via regulation of clock transcription. Current Biology 24: 1248-1255. doi: 10.1016/j.cub.2014.04.018.

Dalle S, Longuet C, Costes S, Broca C, Faruque O, Fontés G, Hani EH, Bataille D. 2004. Glucagon promotes cAMPresponse element-binding protein phosphorylation via activation of ERK1/2 in MIN6 cell line and isolated islets of Langerhans. The Journal of Biological Chemistry 279:20345-20355. doi: 10.1074/jbc.M312483200.

Damiola F, Le Minh N, Preitner N, Kornmann B, Fleury-Olela F, Schibler U. 2000. Restricted feeding uncouples circadian oscillators in peripheral tissues from the central pacemaker in the suprachiasmatic nucleus. Genes \& Development 14:2950-2961. doi: 10.1101/gad.183500.

Dibner C, Schibler U, Albrecht U. 2010. The mammalian circadian timing system: organization and coordination of central and peripheral clocks. Annual Review of Physiology 72:517-549. doi: 10.1146/annurev-physiol-021909135821.

Drucker DJ. 2005. Biologic actions and therapeutic potential of the proglucagon-derived peptides. Nature Clinical Practice Endocrinology \& Metabolism 1:22-31. doi: 10.1038/ncpendmet0017.

Dunlap JC. 1999. Molecular bases for circadian clocks. Cell 96:271-290. doi: 10.1016/S0092-8674(00)80566-8.

Dunphy JL, Taylor RG, Fuller PJ. 1998. Tissue distribution of rat glucagon receptor and GLP-1 receptor gene expression. Molecular and Cellular Endocrinology 141:179-186. doi: 10.1016/S0303-7207(98)00096-3.

Golombek DA, Rosenstein RE. 2010. Physiology of circadian entrainment. Physiological Reviews 90:1063-1102. doi: 10.1152/physrev.00009.2009.

Gonzalez GA, Montminy MR. 1989. Cyclic AMP stimulates somatostatin gene transcription by phosphorylation of CREB at serine 133. Cell 59:675-680. doi: 10.1016/0092-8674(89)90013-5.

Gros L, Thorens B, Bataille D, Kervran A. 1993. Glucagon-like peptide-1-(7-36) amide, oxyntomodulin, and glucagon interact with a common receptor in a somatostatin-secreting cell line. Endocrinology 133:631-638. doi: 10.1210/endo.133.2.8102095.

Hatori M, Vollmers C, Zarrinpar A, DiTacchio L, Bushong EA, Gill S, Leblanc M, Chaix A, Joens M, Fitzpatrick JA, Ellisman MH, Panda S. 2012. Time-restricted feeding without reducing caloric intake prevents metabolic diseases in mice fed a high-fat diet. Cell Metabolism 15:848-860. doi: 10.1016/j.cmet.2012.04.019.

Herichova I. 2013. Changes of physiological functions induced by shift work. Endocrine Regulations 47:159-170. doi: 10.4149/endo_2013_03_159.

Hirao A, Tahara Y, Kimura I, Shibata S. 2009. A balanced diet is necessary for proper entrainment signals of the mouse liver clock. PLOS ONE 4:e6909. doi: 10.1371/journal.pone.0006909.

Jorgensen R, Kubale V, Vrecl M, Schwartz TW, Elling CE. 2007. Oxyntomodulin differentially affects glucagon-like peptide-1 receptor beta-arrestin recruitment and signaling through Galpha(s). The Journal of Pharmacology and Experimental Therapeutics 322:148-154. doi: 10.1124/jpet.107.120006.

Kornmann B, Preitner N, Rifat D, Fleury-Olela F, Schibler U. 2001. Analysis of circadian liver gene expression by ADDER, a highly sensitive method for the display of differentially expressed mRNAs. Nucleic Acids Research 29: E51. doi: 10.1093/nar/29.11.e51.

Lamia KA, Storch KF, Weitz CJ. 2008. Physiological significance of a peripheral tissue circadian clock. Proceedings of the National Academy of Sciences of USA 105:15172-15177. doi: 10.1073/pnas.0806717105.

Le Quellec A, Kervran A, Blache P, Ciurana AJ, Bataille D. 1992. Oxyntomodulin-like immunoreactivity: diurnal profile of a new potential enterogastrone. The Journal of Clinical Endocrinology and Metabolism 74:1405-1409.

LeSauter J, Hoque N, Weintraub M, Pfaff DW, Silver R. 2009. Stomach ghrelin-secreting cells as food-entrainable circadian clocks. Proceedings of the National Academy of Sciences of USA 106:13582-13587. doi: 10.1073/pnas. 0906426106.

Lowden A, Moreno C, Holmback U, Lennernas M, Tucker P. 2010. Eating and shift work - effects on habits, metabolism and performance. Scandinavian Journal of Work, Environment \& Health 36:150-162. doi: 10.5271/sjweh.2898.

Lowrey PL, Takahashi JS. 2004. Mammalian circadian biology: elucidating genome-wide levels of temporal organization. Annual Review of Genomics and Human Genetics 5:407-441. doi: 10.1146/annurev.genom.5. 061903.175925 
Mayo KE, Miller LJ, Bataille D, Dalle S, Göke B, Thorens B, Drucker DJ. 2003. International Union of Pharmacology. XXXV. The glucagon receptor family. Pharmacological Reviews 55:167-194. doi: 10.1124/pr.55.1.6.

Miller BH, McDearmon EL, Panda S, Hayes KR, Zhang J, Andrews JL, Antoch MP, Walker JR, Esser KA, Hogenesch JB, Takahashi JS. 2007. Circadian and CLOCK-controlled regulation of the mouse transcriptome and cell proliferation. Proceedings of the National Academy of Sciences of USA 104:3342-3347. doi: 10.1073/pnas. 0611724104.

Oike H, Nagai K, Fukushima T, Ishida N, Kobori M. 2011. Feeding cues and injected nutrients induce acute expression of multiple clock genes in the mouse liver. PLOS ONE 6:e23709. doi: 10.1371/journal.pone.0023709.

Oster H, Yasui A, van der Horst GT, Albrecht U. 2002. Disruption of mCry2 restores circadian rhythmicity in mPer2 mutant mice. Genes \& Development 16:2633-2638. doi: 10.1101/gad.233702.

Panjwani N, Mulvihill EE, Longuet C, Yusta B, Campbell JE, Brown TJ, Streutker C, Holland D, Cao X, Baggio LL, Drucker DJ. 2013. GLP-1 receptor activation indirectly reduces hepatic lipid accumulation but does not attenuate development of atherosclerosis in diabetic male ApoE(-/-) mice. Endocrinology 154:127-139. doi: 10.1210/en. 2012-1937.

Pfaffl MW. 2001. A new mathematical model for relative quantification in real-time RT-PCR. Nucleic Acids Research 29:e45. doi: 10.1093/nar/29.9.e45.

Rosenberg R, Doghramji PP. 2011. Is shift work making your patient sick? Emerging theories and therapies for treating shift work disorder. Postgraduate Medicine 123:106-115. doi: 10.3810/pgm.2011.09.2465.

Salgado-Delgado R, Angeles-Castellanos M, Saderi N, Buijs RM, Escobar C. 2010. Food intake during the normal activity phase prevents obesity and circadian desynchrony in a rat model of night work. Endocrinology 151 : 1019-1029. doi: 10.1210/en.2009-0864.

Scrocchi LA, Brown TJ, MaClusky N, Brubaker PL, Auerbach AB, Joyner AL, Drucker DJ. 1996. Glucose intolerance but normal satiety in mice with a null mutation in the glucagon-like peptide 1 receptor gene. Nature Medicine 2: 1254-1258. doi: 10.1038/nm1196-1254.

Sinclair EM, Yusta B, Streutker C, Baggio LL, Koehler J, Charron MJ, Drucker DJ. 2008. Glucagon receptor signaling is essential for control of murine hepatocyte survival. Gastroenterology 135:2096-2106. doi: 10.1053/j. gastro.2008.07.075.

Stanley S, Wynne K, McGowan B, Bloom S. 2005. Hormonal regulation of food intake. Physiological Reviews 85: 1131-1158. doi: 10.1152/physrev.00015.2004.

Stokkan KA, Yamazaki S, Tei H, Sakaki Y, Menaker M. 2001. Entrainment of the circadian clock in the liver by feeding. Science 291:490-493. doi: 10.1126/science.291.5503.490.

Sun X, Dang F, Zhang D, Yuan Y, Zhang C, Wu Y, Wang Y, Liu Y. 2015. Glucagon-CREB/CRTC2 signaling cascade regulates hepatic BMAL1 protein. The Journal of Biological Chemistry 290:2189-2197. doi: 10.1074/jbc.M114. 612358.

Tahara Y, Otsuka M, Fuse Y, Hirao A, Shibata S. 2011. Refeeding after fasting elicits insulin-dependent regulation of Per2 and Rev-erbalpha with shifts in the liver clock. Journal of Biological Rhythms 26:230-240. doi: 10.1177/ 0748730411405958.

Takahashi JS, Hong HK, Ko CH, McDearmon EL. 2008. The genetics of mammalian circadian order and disorder: implications for physiology and disease. Nature Reviews Genetics 9:764-775. doi: 10.1038/nrg2430.

Tang-Christensen M, Vrang N, Larsen PJ. 2001. Glucagon-like peptide containing pathways in the regulation of feeding behaviour. International Journal of Obesity and Related Metabolic Disorders 25(Suppl 5):S42-S47. doi: 10.1038/sj.ijo.0801912.

Vollmers C, Gill S, DiTacchio L, Pulivarthy SR, Le HD, Panda S. 2009. Time of feeding and the intrinsic circadian clock drive rhythms in hepatic gene expression. Proceedings of the National Academy of Sciences of USA 106: 21453-21458. doi: 10.1073/pnas.0909591106.

Welsh DK, Takahashi JS, Kay SA. 2010. Suprachiasmatic nucleus: cell autonomy and network properties. Annual Review of Physiology 72:551-577. doi: 10.1146/annurev-physiol-021909-135919.

Yamajuku D, Inagaki T, Haruma T, Okubo S, Kataoka Y, Kobayashi S, Ikegami K, Laurent T, Kojima T, Noutomi K, Hashimoto S, Oda H. 2012. Real-time monitoring in three-dimensional hepatocytes reveals that insulin acts as a synchronizer for liver clock. Scientific Reports 2:439. doi: 10.1038/srep00439.

Yan L, Silver R. 2002. Differential induction and localization of mPer1 and mPer2 during advancing and delaying phase shifts. The European Journal of Neuroscience 16:1531-1540. doi: 10.1046/j.1460-9568.2002.02224.x.

Yan J, Wang H, Liu Y, Shao C. 2008. Analysis of gene regulatory networks in the mammalian circadian rhythm. PLOS Computational Biology 4:e1000193. doi: 10.1371/journal.pcbi.1000193.

Yoo SH, Yamazaki S, Lowrey PL, Shimomura K, Ko CH, Buhr ED, Siepka SM, Hong HK, Oh WJ, Yoo OJ, Menaker M, Takahashi JS. 2004. PERIOD2::LUCIFERASE real-time reporting of circadian dynamics reveals persistent circadian oscillations in mouse peripheral tissues. Proceedings of the National Academy of Sciences of USA $101:$ 5339-5346. doi: 10.1073/pnas.0308709101. 